\title{
Voigt profile introduces optical depth dependent systematic errors - detected in high resolution laboratory spectra of water
}

\author{
Authors: Manfred Birk ${ }^{1 *}$, Georg Wagner ${ }^{1}$
}

\author{
Affiliations: \\ ${ }^{1}$ Deutsches Zentrum für Luft- und Raumfahrt e.V. (DLR), Remote Sensing Technology Institute, \\ Oberpfaffenhofen, D-82234 Wessling, Germany. \\ *Correspondence to: Manfred Birk, Manfred.birk@dlr.de
}

\begin{abstract}
:
The Voigt profile commonly used in radiative transfer modeling of Earth's and planets' atmospheres for remote sensing/climate modeling produces systematic errors so far not accounted for. Saturated lines are systematically too narrow when calculated from pressure broadening parameters based on the analysis of laboratory data with the Voigt profile. This is caused by line narrowing effects leading to systematically too small fitted broadening parameters when applying the Voigt profile. These effective values are still valid to model non-saturated lines with sufficient accuracy. Saturated lines dominated by the wings of the line profile are sufficiently accurately modeled with a Voigt profile with the correct broadening parameters and are thus systematically too narrow when calculated with the effective values. The systematic error was quantified by mid infrared laboratory spectroscopy of the water $v_{2}$ fundamental. Correct Voigt profile based pressure broadening parameters for saturated lines were 3-4\% larger than the effective ones in the spectroscopic database. Impacts on remote sensing and climate modeling are expected. Combination of saturated and non-saturated lines in the spectroscopic analysis will quantify line narrowing with unprecedented precision.
\end{abstract}

Keywords: Voigt line profile, speed-dependent Voigt line profile, radiative transfer, optical depth, systematic errors

\section{Introduction}

Clear sky radiative transfer of the Earth's atmosphere from far to near infrared spectral region is used in climate modeling [1] and remote sensing (e.g.[2][3][4]) and requires a line-by-line spectroscopic database such as HITRAN [5] or GEISA [6]. The calculation of atmospheric signatures is usually based on the Voigt line profile [7] which is a convolution of Doppler and Lorentz broadening profiles. In laboratory spectroscopy the Voigt profile is also widely used to deduce spectroscopic parameters such as pressure broadened widths and line intensities. The 
Voigt profile is known to be an approximation disregarding collisional and speed-dependent narrowing effects [8] relevant in certain pressure and temperature regimes. The residuals in Voigt line fitting of laboratory spectra are in case of water (total pressure $200-1000$ mbar) ca. $\pm 0.5 \%$ of peak absorption for air broadening parameters $>0.06 \mathrm{~cm}^{-1} / \mathrm{atm}$, as modelled based on the data set used in this work. These broad lines are strong and dominate the spectra. The residuals get larger in case of narrow, usually weaker, lines with pressure broadening parameters $<0.02 \mathrm{~cm}^{-1}$.

As an example, the largest residuals found in spectra recorded in the DLR spectroscopic laboratory resulting from a Voigt fit of an air-broadened water line are shown in Figure 1. The typical W-shaped residuals have amplitudes of $\pm 2.5 \%$ with respect to the peak absorption. The Voigt fit of an air-broadened line yields a nearly correct line intensity (typical errors ca. $0.5 \%$ for strong lines, worst case up to 3\%) approximately represented by the area under the non-opaque spectrum. Thus, if such an optically thin line is measured in the atmosphere the number density retrieved using Voigt parameters will be nearly correct. The small line profile differences to the more sophisticated profiles near the line center are furthermore spectrally smeared out by the mostly coarse resolution of remote sensing instruments. Therefore, systematic errors of radiative transfer due to this approximation should be negligible at first glance.

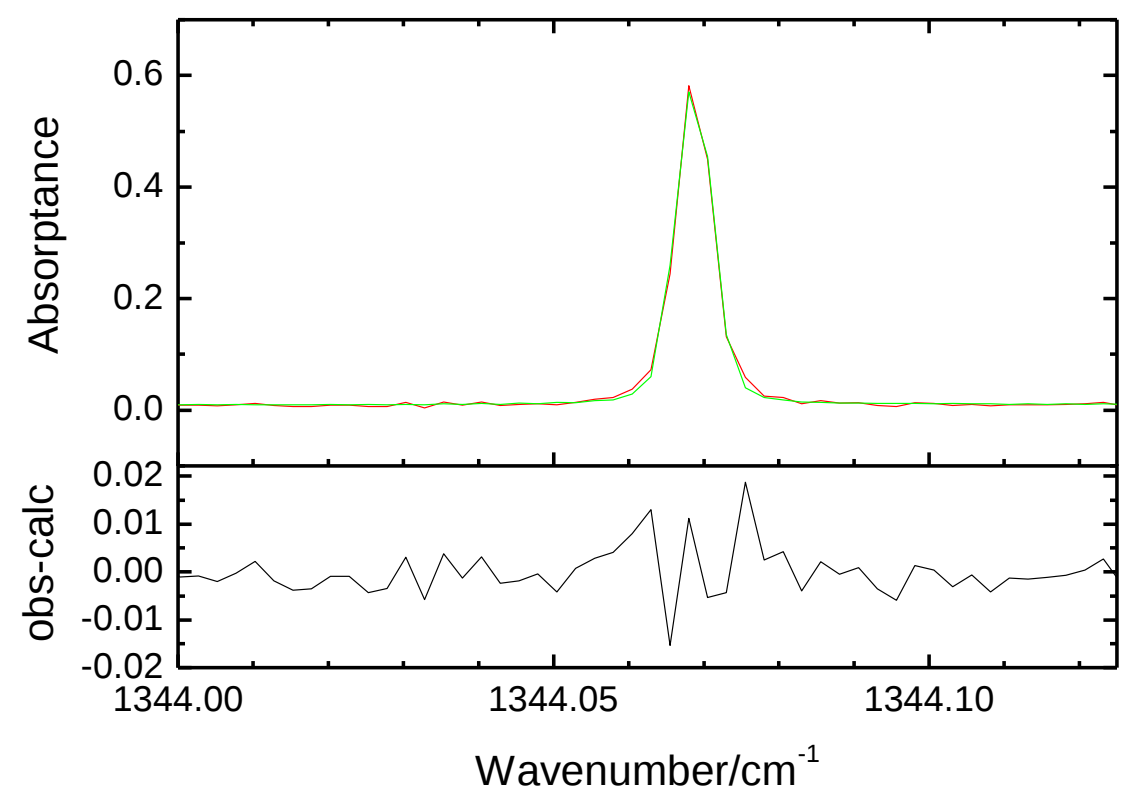

Figure 1. Voigt fit of line showing one of the largest observed W-shaped residuals in our measurements. Experiment described in [9], measurement details: T $317 \mathrm{~K}, P_{\text {tot }} 50.43$ mbar, $P_{\text {H2O }}$ 0.2159 mbar, absorption path $7862.7 \mathrm{~cm}$, maximum optical path difference $187.5 \mathrm{~cm}$.

Spectral residuals due to line narrowing have only been observed in up-looking high resolution Fourier-transform observations of HF in solar occultation [10][11]. In one other case (up-looking observations of $\mathrm{H}_{2} \mathrm{O}$ ) also a non-Voigt profile (Galatry) was used [12]. An improvement in 
residuals in comparison to the Voigt profile was not observed but the resulting atmospheric trace gas profiles matched better with validation sources. There seems not to have been an urgent need to use more sophisticated line profiles than Voigt for remote sensing. Narrowing parameters are rather rare in literature and no entries are routinely available in HITRAN or GEISA.

In order to introduce the problem addressed in this paper the limitation of line parameters should be illuminated. Line parameters are based on a physical model and thus extrapolation should be feasible. However, any model error will cause systematic errors for extrapolation which are hard to predict. The problem described in the following is associated with extrapolation in optical depth.

\section{Problems with opaque lines}

Laboratory high resolution Fourier-transform measurements of the air-broadened water $v_{2}$ fundamental band around $7 \mu \mathrm{m}$ were carried out at DLR. The aim of the work was to provide spectroscopic data with high and defined accuracy. Line intensities have been analyzed [13] and were included in the HITRAN2008 database [5]. Recently, temperature-dependent air broadening parameters were published based on the Voigt profile [9] and were included in HITRAN2012 [15]. Details on the experiment used for line intensity and broadening analysis are given in [9]. Numerous measurements were recorded covering four orders of magnitude in $\mathrm{H}_{2} \mathrm{O}$ column density. Thus, many transitions occurred in several spectra with optical depth, $O$, ranging from optically thin to saturated. An example can be seen in Figure 2. Microwindows in single spectra were fitted applying the Voigt model, resulting in line positions, line intensities, and Lorentz widths for each measurement. Lines with peak optical depth $>4$ (transmittance $<1.8 \%$ ) were excluded from further analysis because of the high correlation between line intensity and line broadening at high optical depth. Furthermore, optically thick lines show a higher susceptibility of line parameters to systematic errors like detector non-linearity or instrumental line shape errors. It should be noted that omitting opaque lines from the line parameter analysis is a very common procedure. The maximum peak optical depth encountered was about 10000 in case of 200 mbar air-broadened measurements. In a further analysis the spectroscopic parameters from the single measurements were merged, resulting in line positions, pressure-induced shifts with temperature dependence, line intensities and self- and air-broadening parameters with temperature dependence. Several tests were dedicated to the validation of data product accuracy. In one test air broadening parameters merged from different peak optical depth ranges $(O<0.5$, $0.5<O<4$ ) were compared. On average, the air broadening parameters from lines of higher opacity were larger by $0.57(6) \%$. Since this difference is rather small it was concluded that there is no optical depth dependence of the air broadening. 


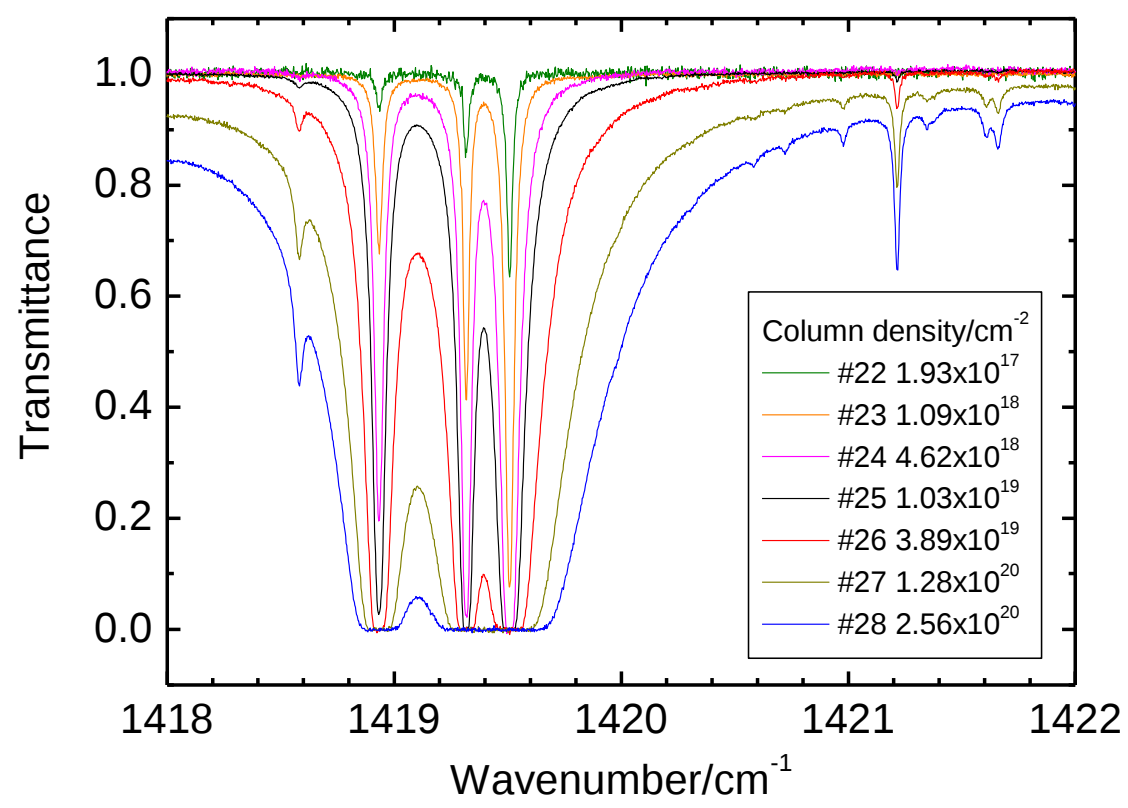

Figure 2. Example of a microwindow showing the large dynamic range of column density and optical depth.

An important quality check of a new spectroscopic database [9][13] is to reproduce the measured spectra used in the analysis within the noise level. In a first step this is done only for spectral regions with spectral features used in the analysis. Thus, regions with opaque spectral features are excluded. In order to use these regions for quality check several requirements have to be fulfilled:

- Accurate line intensities and broadening parameters with defined uncertainties (correlation!)

- Good knowledge of $0 \%$ and 100\% level, e.g. good reference spectra, no channeling, non-linearity correction if necessary

- Good knowledge of instrumental line shape function

- Assessment of self broadening contribution to line width

For most of the published spectroscopic work not all of these requirements are fulfilled and thus the opaque spectral regions were not used. In the present case, however, intensities and air broadening parameters with defined uncertainties were available even from the same source and all other points were also taken into account. It must be admitted that it took several iterations including an effective Hamiltonian approach for the line intensities to reach the status to use opaque regions for quality check. There was an additional motivation arising from the statement by S.A. Clough: “valleys between strong lines in IASI spectra are modeled too deep” [16]. 
The quality check of optically thin lines mostly shows no residuals. An example is given in Figure 3A. In contrast optically thick lines systematically showed prominent residuals, as can be seen in Figure 3B. The scale of OMC in A was expanded by 2 with respect to B to account for the $50 \%$ peak absorption in A vs $100 \%$ in B. The detection limit for W-shaped residuals is about $0.5 \%$ of the line peak. The residuals can be significantly reduced by fitting the air broadening width as indicated in the red trace in Figure 3B. This was done for several opaque lines and the fitted air broadening width was found to be $3-4 \%$ larger when compared to those published in [9].

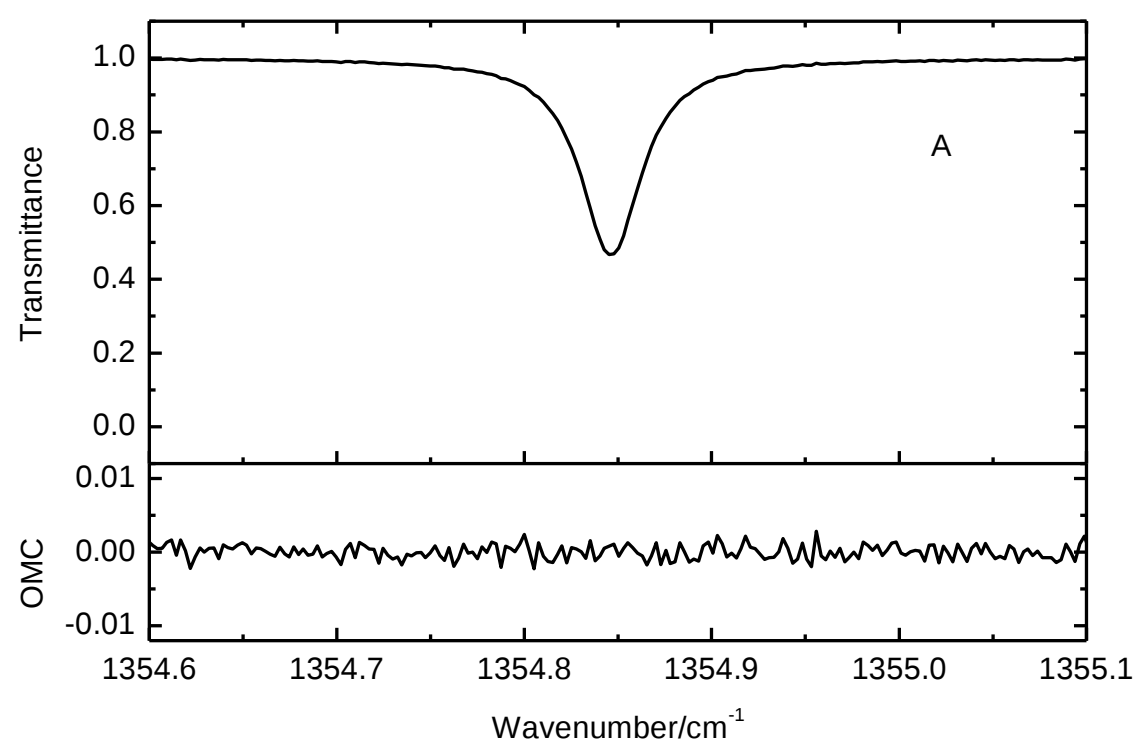




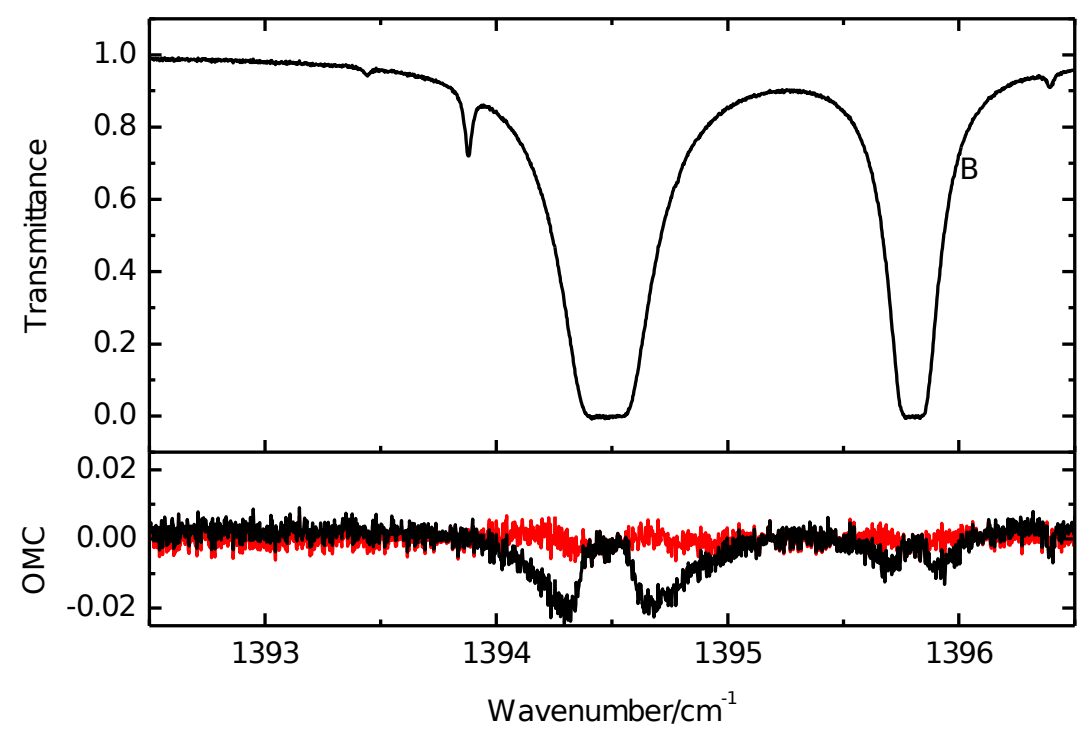

Figure 3. Experimental spectrum (A: $P_{\text {H2O }} 0.2$ mbar, B: $P_{\text {H2O }} 2.5$ mbar, $P_{\text {tot }}\left(\mathrm{H}_{2} \mathrm{O}+\right.$ air) 200 mbar, absorption path $21 \mathrm{~m}$, temperature $296 \mathrm{~K}$, instrumental resolution $0.0027 \mathrm{~cm}^{-1}$ ) in upper panels. Spectrum modeled using Voigt lineshape with spectroscopic data from [9][13], lower panels observed-calculated (OMC). A: non-saturated line, OMC shows only noise. B: saturated lines, clearly visible differences from noise (black curve) indicating that the spectroscopic data are not appropriate in this case. The residuals can be significantly reduced by fitting the air broadening width (red curve).

\section{Explanation}

The reason for the residuals of saturated lines as shown in Figure 3 was found to be an undiscovered error propagation associated with the Voigt lineshape. An optical depth dependent error in combination with line parameters and their associated models was to our knowledge never considered. Therefore the extrapolation regarding optical depth was ignored.

It is useful to recall the dependence of transmittance and optical depth on line parameters. Transmittance spectra and optical depth are linked by the following equation

$$
\tau=\exp \left(-\alpha\left(T, P_{k}, \sigma\right) \cdot l \cdot N\right)=\exp (-O)
$$

with transmittance $\tau$, optical depth $O$, absorption cross section $\alpha$, temperature $T$, pressure $P$, wavenumber $\sigma$, absorption path $l$, number density of absorbing gas $N$. $k$ is the index of different gases in the mixture, e.g. $P_{H 2 O}$ and $P_{\text {air }}$. The terms "saturated", "large optical depth", and "optically thick" are equivalent and refer to the line center. There is no clear definition for the usage of these terms, however, in the present paper optical depth $>4$ qualifies as saturated. The absorption cross section can be expressed in terms of line parameters. 


$$
\alpha=\sum_{i} S_{i}(T) \cdot f\left(T, P_{k}, \sigma-\sigma_{0 i}+\sigma_{s i k}, \gamma_{i k}, n_{i k}, \ldots\right)
$$

with monochromatic line profile $f$, e.g. the Voigt profile, line index $i$, line position $\sigma_{0}$, line intensity $S$, pressure-induced line shift $\sigma_{s}$, pressure broadening parameter $\gamma$, temperature exponent $n$, and further parameters, e.g. narrowing parameter, temperature dependence of pressure-induced line shift. Line parameters currently in the HITRAN database [5] are $\sigma_{0}, S(296$ $\mathrm{K}), \gamma_{\text {air }}(296 \mathrm{~K}), \gamma_{\text {self }}(296 \mathrm{~K}), \sigma_{\mathrm{s}, \text { air }}(296 \mathrm{~K}), n_{\text {air }}$, and the lower state energy $E_{\text {lower }}$ needed to convert the line intensity from $296 \mathrm{~K}$ to the desired temperature $T$. Total internal partition sums which are also required for line intensity conversion are given on an isotopologue basis.

The essential point is the non-linear transformation of the line profile by the exponential function. In the optically thin case the exponential function can be expanded in a power series truncated after the linear term. Thus, the transmittance line profile is can be approximated by the optical depth line profile. The optical depth line profile only scales with column density $\mathrm{Nl}$ but does not change the shape. The transmittance line profile, however, changes markedly for increasing peak optical depth. With increasing peak optical depth the region close to line center with transmittance values close to zero becomes wider. Thus, the central part of the line profile function is masked out. This can be quantified by the derivative of the transmittance with respect to optical depth $(d \tau / d O)\left(\sigma-\sigma_{0}\right)$ as function of the distance to the line center $\sigma-\sigma_{0}$. The maximum is at $O=1$, close to the distance to line center for $50 \%$ of peak absorption, $\sigma_{a b 550 \%}-\sigma_{0}$.

Figure 4 shows the derivatives for two different peak optical depths for a line with Lorentz HWHM $0.08 \mathrm{~cm}^{-1}$. With increasing peak optical depth the masked out region close to the line center grows. For a peak optical depth of 800 or 4500 the maximum contribution is at 2 and $5.5 \mathrm{~cm}^{-1}$, respectively, which is in the far line wing - 25 and 70 HWHMs away from line center. In Figure $5 \sigma_{a b 550 \%}-\sigma_{0}$ is plotted vs. the peak optical depth. The maximum peak optical depth in Figure 5 is ca. $10^{6}$ corresponding to the optical depth for the strongest $\mathrm{H}_{2} \mathrm{O} v_{2}$ line $\left(\mathrm{S}=3.2 \times 10^{-19}\right.$ $\mathrm{cm}^{-1}$ (molecule $\left.\mathrm{cm}^{-2}\right)$ ) for an airmass 2 atmospheric water column (AFGL tropical [14]). The corresponding distance is about $100 \mathrm{~cm}^{-1}$ which is far beyond the $25 \mathrm{~cm}^{-1}$ cut-off used for continuum determination [17]. In this extreme far wing situation the value of the Lorentz profile is only $10^{-6}$ of its maximum.

These considerations have two consequences:

1. Model errors in the line profile will propagate differently for opaque and optically thin lines since different parts of the line profile are important.

2. Optical depth can be used to focus on a certain section of the line profile and to mask out the center part. Thus, opaque lines are excellent for investigating line wing effects.

Using pressure broadening data retrieved from lines with peak optical depth $<4$ for peak optical depth $>4$ is an extrapolation with potential errors if the line model is not sufficiently accurate. A distinct error of the Voigt profile is neglecting effects as Dicke [18] and speed-dependent [19][20] narrowing. Figure 6 shows the ratio of a SDVP using the quadratic model for speed dependence of collisional width [21] and a Voigt profile, calculated with the 
same air broadening parameter. It can be seen that the profiles are different in the line center but agree in the line wings. Thus, for sufficiently opaque lines $(\mathrm{O}>4)$, masking out the line center, the effect of narrowing is not relevant. Thus, opaque lines can be approximated by a pure Lorentzian profile. A problem occurs when the Lorentzian width is obtained by an effective Voigt fit of non-opaque narrowed lines. In this case the narrowing leads to a systematically too small Lorentzian width which is also too small for opaque lines which require the correct Lorentzian width.

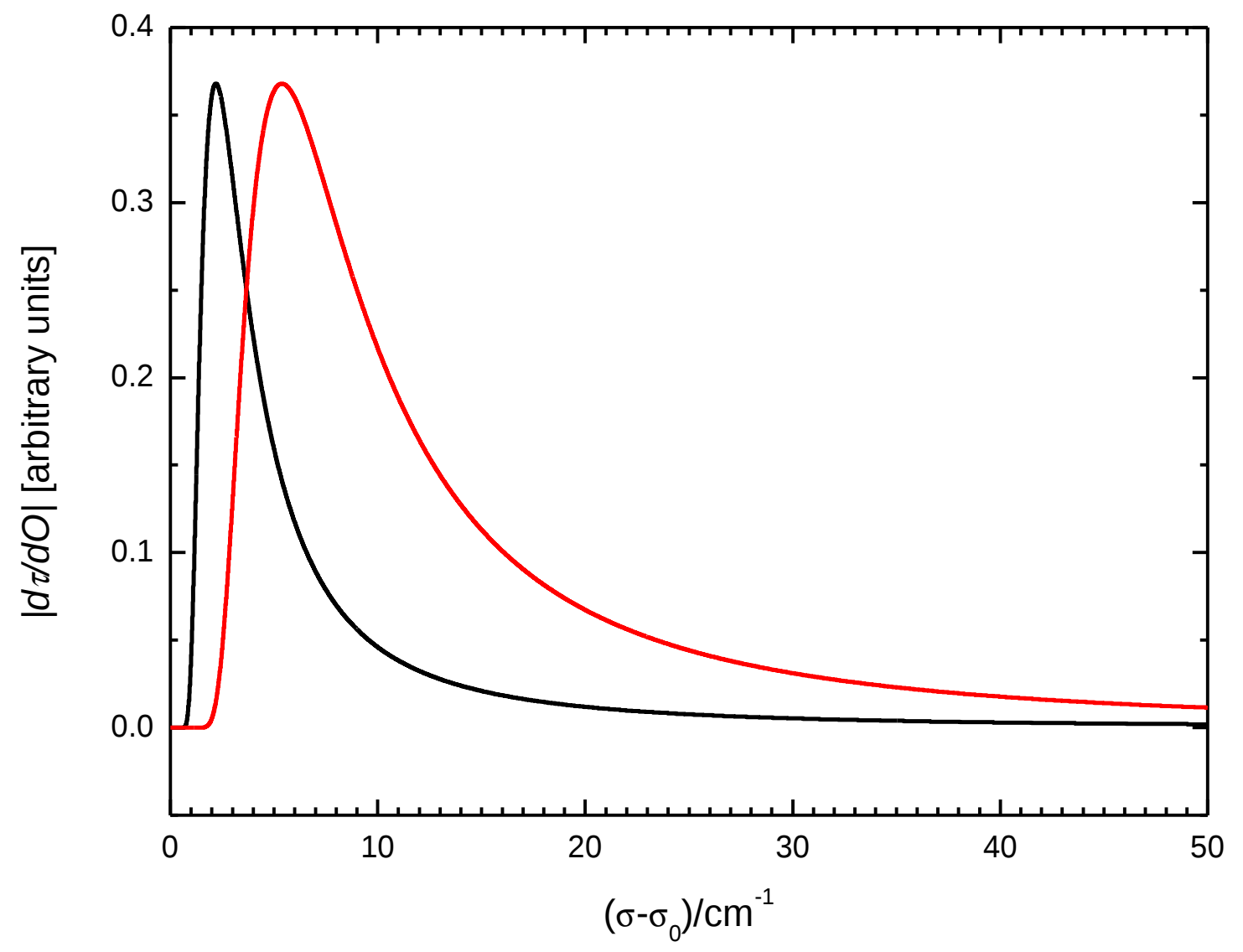

Figure 4. Derivative of transmittance with respect to optical depth as function of distance to line center for a Lorentzian line with FWHM $0.08 \mathrm{~cm}^{-1}$. Black: peak optical depth 800, red: peak optical depth 4500. 


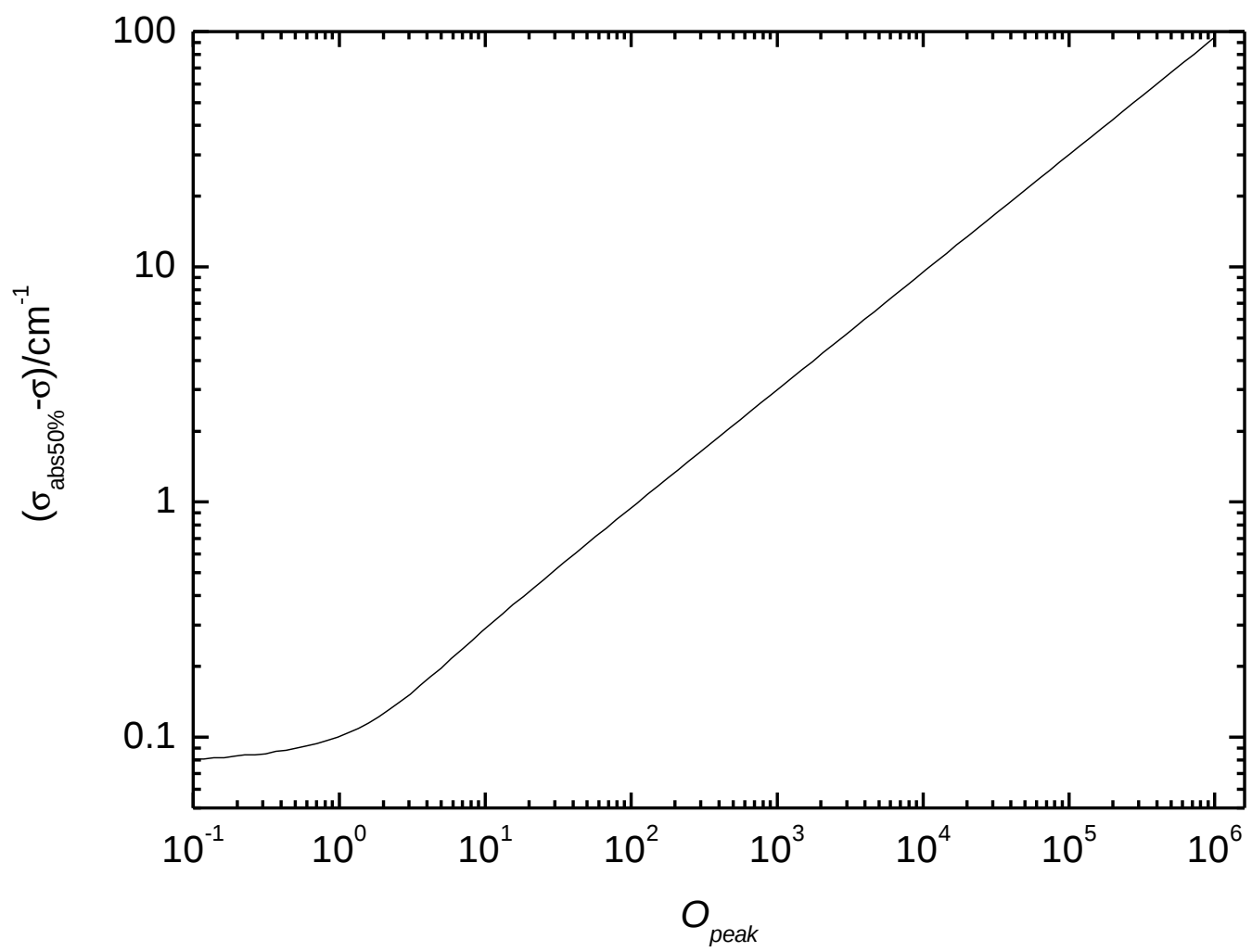

Figure 5. Distance to line center for 50\% of peak absorption as function of peak optical depth.

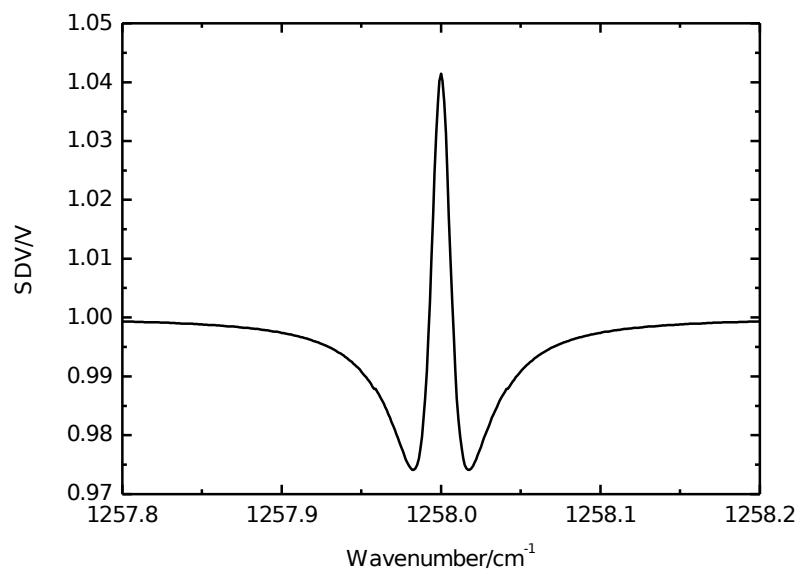

Figure 6. Ratio of SDVP and Voigt lineshapes (air broadening parameter $0.063 \mathrm{~cm}^{-1} / \mathrm{atm}$, narrowing parameter $0.012 \mathrm{~cm}^{-1} / \mathrm{atm}, P_{\text {tot }} 200 \mathrm{mbar}$ ). It can be clearly seen that the lineshapes approach each other in the line wings. 
Figure 7 shows a SDVP and Voigt profile calculated for the same line position, intensity, and air broadening parameter in case of a non-saturated (A) and a saturated (B) line. The SDVP was modeled with a typical narrowing parameter. Whereas the differences in the non-saturated case are significant (peak-to-peak $6 \%$ of peak absorption) they are small for the saturated case. Figure 7C,D shows an effective Voigt fit of the same speed-dependent Voigt lines. Case C corresponds to the typical laboratory spectroscopy approach fitting non-opaque lines with narrowing using a Voigt profile while case D corresponds to the fit producing the red residuals in Figure 3B. In the non-saturated case the OMC drop by a factor 3 when compared to the difference in Fig. 7A and show the typical M-shape (W-shape in absorption) of an effective Voigt fit. The residuals are so small that they vanish in the noise in case of the experimental spectrum in Fig. 3A. The line narrowing in the speed-dependent Voigt causes the fitted effective Voigt profile based air broadening parameter to be decreased by $4.5 \%$. In case of the saturated line (D) the fitted air broadening parameter is only decreased by $0.6 \%$ and the residuals are small. The chosen case had a moderate optical depth and thus the line center is not completely masked out. Increasing optical depth would further decrease residuals and yield a better agreement for the fitted air broadening parameter in case D. Case E shows the problems visible in Figure 3B (black residuals) when modelling a saturated line with the Lorentzian linewidth obtained by effective Voigt fits of non-opaque lines.
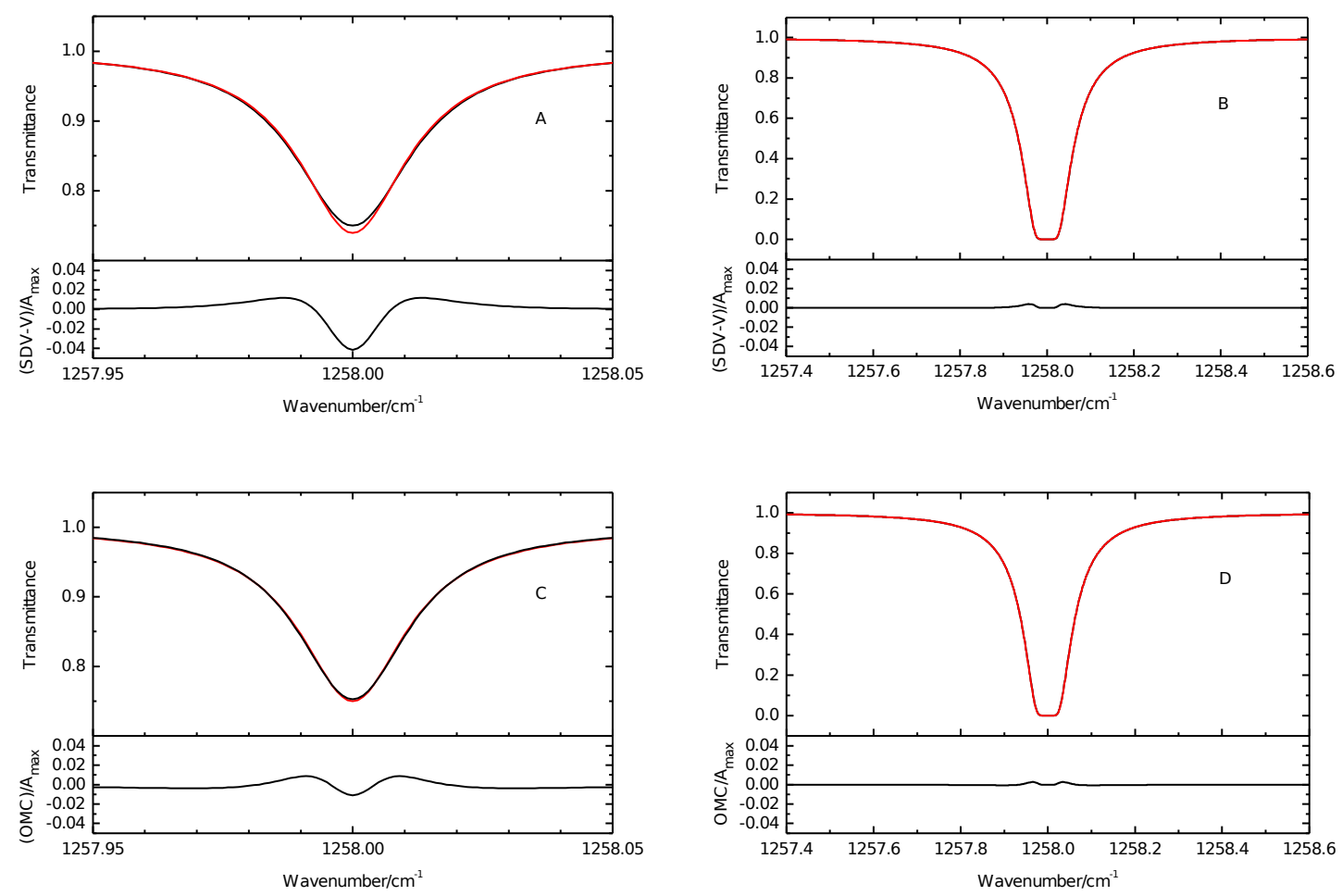


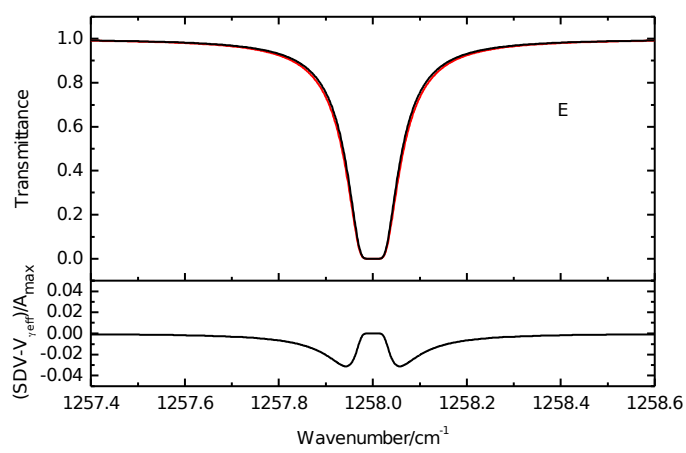

Figure 7. Speed-dependent Voigt: red, Voigt: black. Case A,B: Simulated Voigt and speed-dependent Voigt line with same intensity and Lorentzian linewidth $\left(\gamma_{\text {air }} 0.063 \mathrm{~cm}^{-1} / \mathrm{atm}\right.$, narrowing parameter $0.012 \mathrm{~cm}^{-1} / \mathrm{atm}$ (taken from Fig. 11), $P_{\text {tot }} 200 \mathrm{mbar}$ ). Case A: optically thin line, peak absorption 0.25. Case B: optically thick line, line center optical depth 20. Lower panels: $(S D V-V) / A_{\max }, S D V=$ speed-dependent Voigt, $V=$ Voigt, $A_{\max }=$ peak absorption. Cases $\mathrm{C}$ and D: Same speed-dependent Voigt lines as A and B but Voigt profile fitted to speed-dependent Voigt lines. Case C: fitted line intensity 1.1\% smaller, fitted air broadening parameter $4.5 \%$ smaller. Case D: Line intensity fixed because of correlation with pressure broadening, fitted air broadening parameter $0.6 \%$ smaller. Case E: Same speed-dependent Voigt line as B, but Voigt profile with effective Lorentzian linewidth as fitted from case C.

Although air broadening parameters derived from non-saturated lines are systematically a few percent too low the spectroscopic database reproduces the measured spectrum well (beside small M-shaped residuals mostly buried in the noise). The fitted Voigt air broadening parameters are thus effective values. In case both, laboratory and remote sensing measurements, are based on optically thin lines only, no error in the retrieved atmospheric parameter occurs.

\section{Proof}

In the first step two measurements were selected with different water pressures but same total pressure. In the original analysis [9][13] line intensity, Lorentzian linewidth and line position were fitted for most transitions. Only results for lines with peak optical depth $<4$ were used in the original analysis to yield averaged line intensities and air broadening parameters $\gamma_{\text {airorig. }}$ In the present analysis, line intensities were fixed to the results of the first analysis and Lorentzian linewidths (converted to air broadening parameter $\gamma_{\text {airnew }}$ ) and line positions were fitted for lines with optical depth $>0.5$. Self broadening parameters from HITRAN 2008 were applied. As expected, residuals such as seen in Fig. 3B disappeared. Figure 8 shows two examples of microwindows with opaque lines. Indeed, the Lorentzian function is sufficient to fit these lines with residuals being smaller than the noise level. Optical depths up to 500 were covered. The part of the line profile with largest $d \tau / d O$ (see Figures 4 and 5) is at a distance of about 26 HWHM from the line center where the intensity of the Lorentz function drops to $0.2 \%$ from the maximum. Thus, these strong lines can be sufficiently represented by the Lorentz profile even so 
far away from the line center. Percentage differences $\left(\gamma_{\text {air,orig- }}-\gamma_{\text {airnew }}\right) / \gamma_{\text {airorig }}$ as function of optical depth for one measurement as example are shown in Fig. 9. For optical depth $>4$ the difference is nearly constant with values between -3 and $-4 \%$. The plot shows that a systematic difference also occurs for optical depths $<4$ which smoothly changes from $-3.5 \%$ to $+1 \%$ for optical depth 0 . The original values were determined as weighted mean from lines with optical depths $<4$ [9]. Since systematic errors differ in the optical depth range from 0 to 4 the average optical depth is linked to the systematic error in the original values. The scatter in Fig. 9 is partly caused by different mean optical depths for different lines. Apparently the average optical depth for all lines in Fig. 9 is on the order of 1 where $\left(\gamma_{\text {airorig }}-\gamma_{\text {airnew }}\right) / \gamma_{\text {airorig }}$ is 0 . This explains the value of $+1 \%$ for $O=0$. If the average optical depth in the laboratory work had been close to 0 the entire curve in Fig. 9 should be shifted down by $1 \%$.

The analyses of other measurements except those at 50 mbar total pressure yield similar results. In case of a 50 mbar measurement the differences are $+2 \%$ at 0 optical depth instead of $+1 \%$. 50 mbar measurements were excluded in the original analysis [9] since they showed systematic errors due to line narrowing when compared to measurements at higher pressure. This shows again that systematic errors due to non-Voigt profiles are to lower extent also present among non-saturated lines and that they are more prominent at lower total pressure. The Lorentz broadening parameters obtained here are close to the speed-independent part of the air broadening of the SDVP while those in the spectroscopic databases obtained from Voigt fits of non-saturated lines are effective parameters compensating for narrowing.

The contribution of self broadening to the Lorentzian linewidth is significant since the investigated saturated lines are present in measurements with higher water pressure. For example the estimated contribution of self broadening to the Lorentzian linewidth is ca. 15\% for $2.5 \mathrm{mbar}$ water in 200 mbar air. Since self broadening data from the HITRAN database were used in the analysis, it needs to be shown that the results in Fig. 9 are not caused by systematic errors in the self broadening parameters. The agreement of the results deduced from spectra recorded with 2.5 and 5 mbar $\mathrm{H} 2 \mathrm{O}$, respectively, in 200 mbar air indicates the absence of systematic errors due to self broadening since the influence of self broadening differs by a factor 2. In Section 2 it was mentioned that in [9] air broadening parameters obtained from lines with $0.5<0<4$ were ca. $0.6 \%$ larger than those obtained from lines with $O<0.5$. The $0.6 \%$ are rather small but still systematic and can now be understood. Again, this shows that lines with smaller optical depth dominate the $0.5<O<4$ region. 


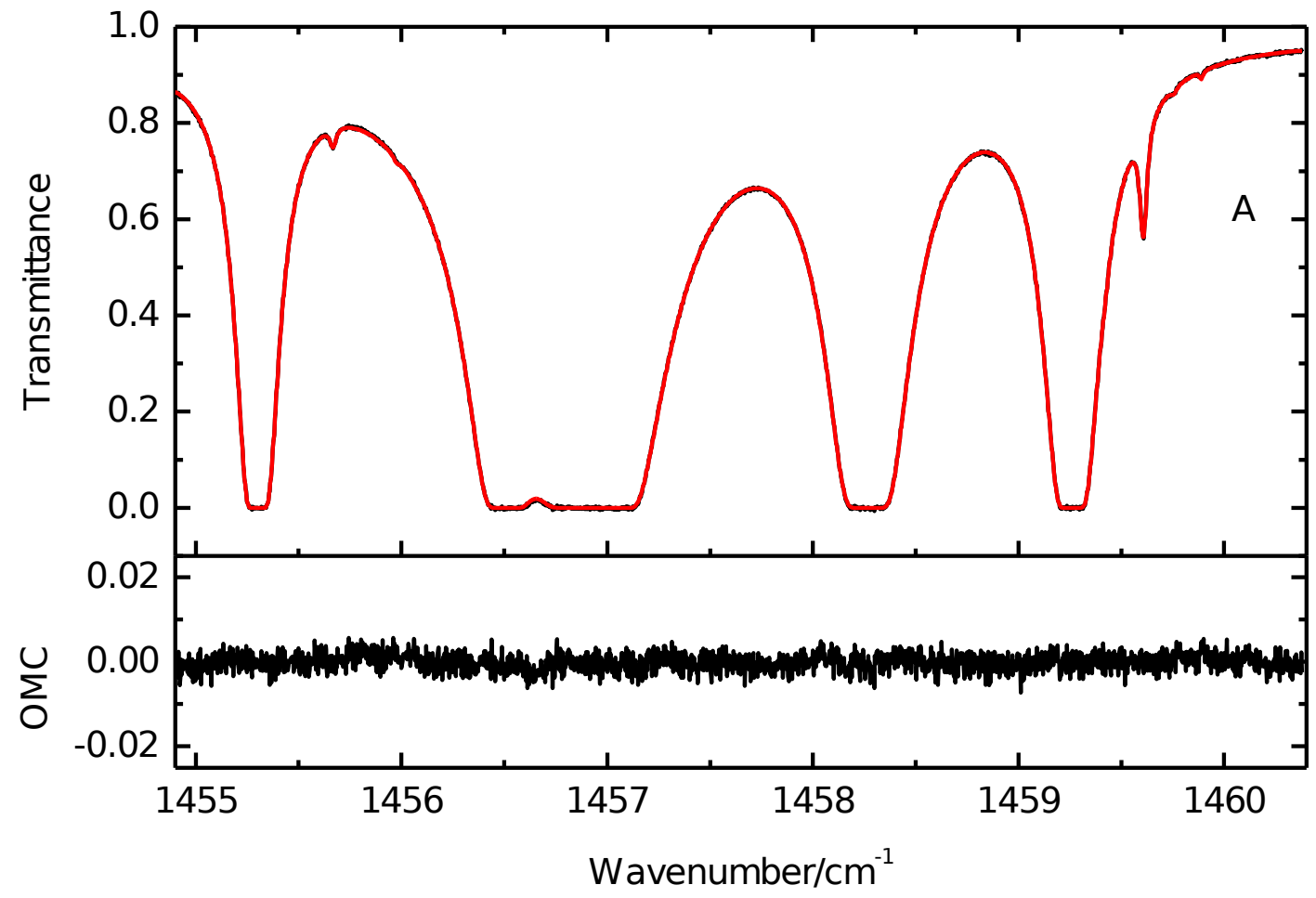




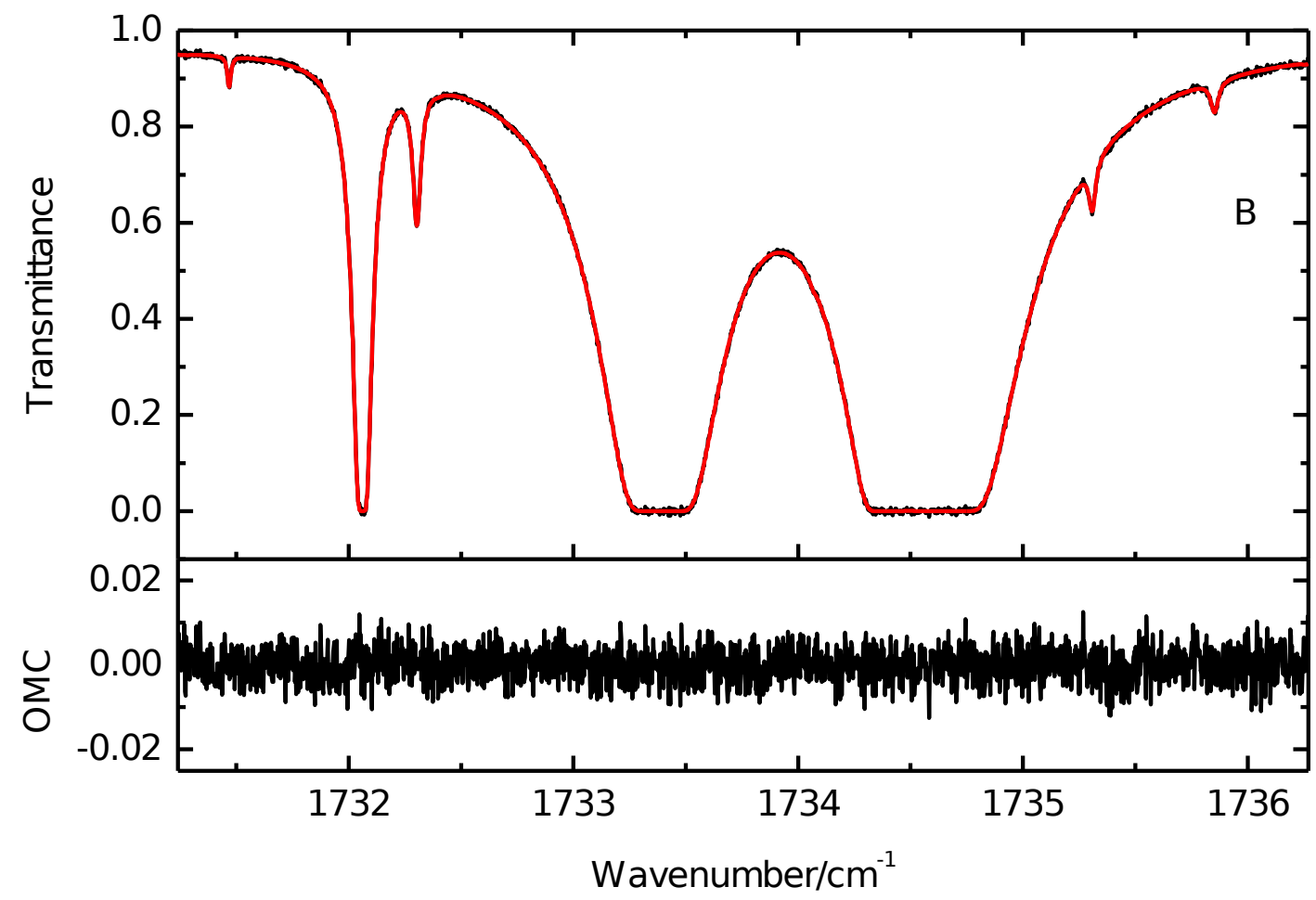

Figure 8. Examples of Voigt fits of opaque lines. Line positions, Lorentz broadening parameters and quadratic baseline were fitted for the saturated lines (Region A: opaque lines position/optical depth $1455.301 \mathrm{~cm}^{-1} / 73,1456.510 \mathrm{~cm}^{-1} / 218,1456.887 \mathrm{~cm}^{-1} / 422,1457.072 \mathrm{~cm}^{-1} / 65,1458.267$ $\mathrm{cm}^{-1} / 143$; region B: $1732.061 \mathrm{~cm}^{-1} / 12,1733.391 \mathrm{~cm}^{-1} / 514,1734.393 \mathrm{~cm}^{-1} / 161,1734.651$ $\mathrm{cm}^{-1} / 303$ ). Observed black, calculated red, observed-calculated in lower panels. Experimental conditions see Fig. 3. 


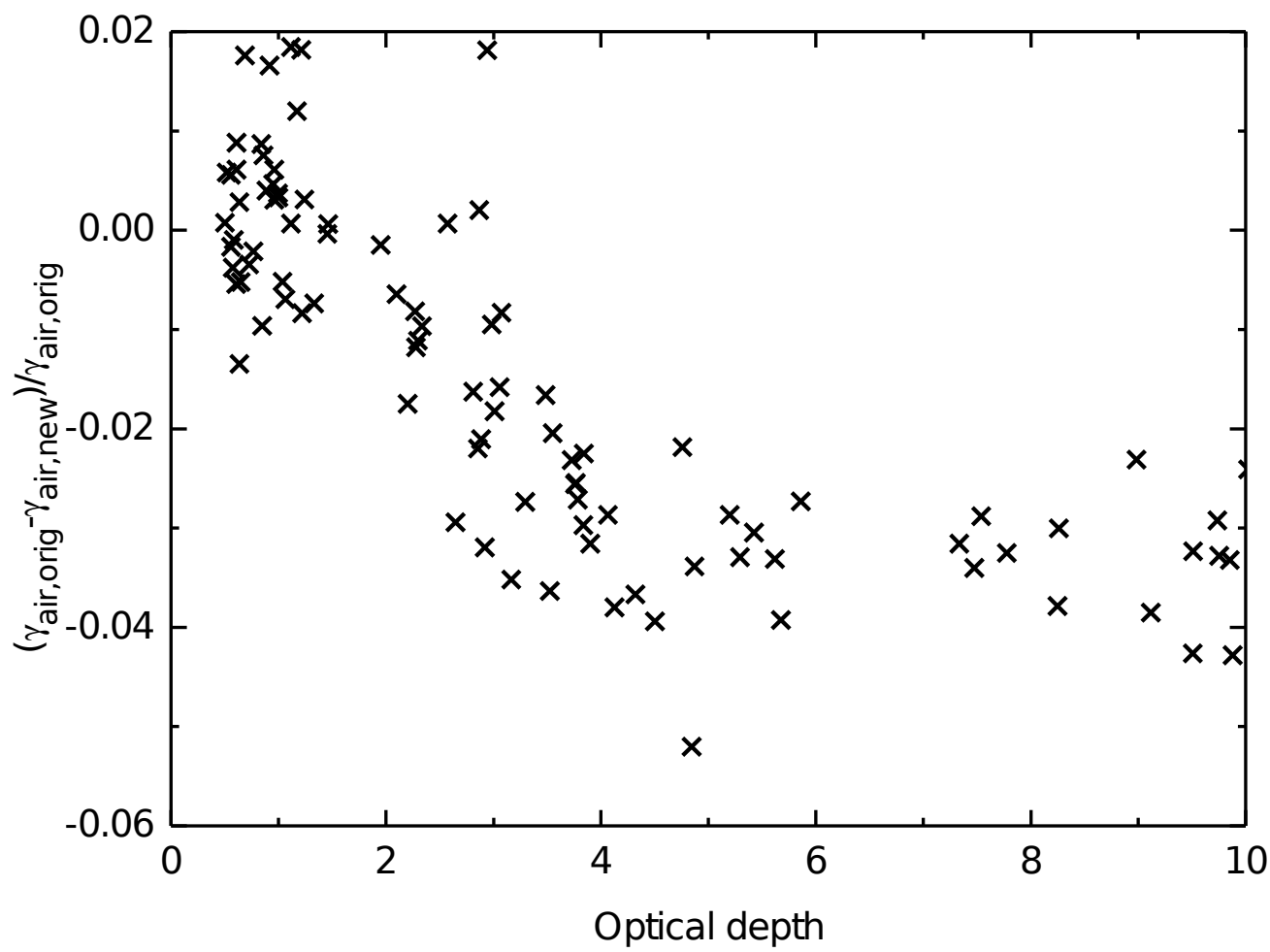

Figure 9. Differences $\left(\gamma_{\text {air,orig- }}-\gamma_{\text {airnew }}\right) / \gamma_{\text {airorig }}$ vs. peak optical depth. Data were available up to optical depth 500, showing differences similar to data between peak optical depths 4 and 10. Only data were displayed with statistical uncertainties $<1 \%$ in $\gamma_{\text {airorig }}$ and $\gamma_{\text {airnew. }}$. Experimental conditions see Fig. 3.

In order to prove that line narrowing indeed causes the difference in original and new pressure broadening parameters the following procedure was carried out. A SDVP [21] was applied to non-saturated lines (measurements selected with 12.5 and 28 times less $\mathrm{H}_{2} \mathrm{O}$ column) to fit line positions, line intensities, and line narrowing, $\gamma_{2}$ air, with the air broadening parameter fixed to the $\gamma_{\text {airnew }}$ values Figure 9 is based on. The precision of the narrowing linewidth was impressive compared to the usual method using the sparse information in the W-shaped residuals. It should be noted that in the line fitting the w-shaped residuals were small and sometimes buried in the noise. Commonly in the literature Lorentzian linewidth and narrowing linewidth are fitted together for non-saturated lines requiring large signal-to-noise ratio and high spectral resolution [22][23][24][25]. In the present case the Lorentzian linewidth is obtained from an independent source, i.e. saturated lines. The residuals of the fits show only noise indicating the validity of the selected line profile function. An example is given in Figure 10. Figure 10A shows the residuals in case $\gamma_{\text {airnew }}$ was used and only baseline, line intensity and position are fitted. This corresponds to Figure 7A. To use $\gamma_{\text {air }}$ from a fit of opaque lines and fix it in a fit of non-opaque lines is the opposite of the usual laboratory approach. While in the usual laboratory approach large residuals appear in case of opaque lines (see Figures 3B and 7E, 
experimental lines broader) here large residuals appear for the non-opaque lines (experimental lines narrower). In Figure 10B an effective Voigt fit was carried out, i.e. $\gamma_{\text {air }}$ was fitted as well. While the left line shows distinct M-shaped residuals the right line shows residuals on the order of the noise. The right line is broader and the ratio of $\gamma_{2}$ air and $\gamma_{\text {air }}$ is much smaller (see arrows in Figure 11). This ratio appears to be linked to the magnitude of the residuals. Figure 10C shows the result of fitting $\gamma_{2 \text { air }}$ while fixing the Lorentz broadening to $\gamma_{\text {airnew. }}$. The residuals being noise proves that the speed-dependent Voigt removes the error of too narrow modelled Voigt lines when extrapolating in optical depth space. Figure 11 shows the fitted narrowing parameters vs. the air broadening parameter for two measurements. The error bars shown in the figure are $1 \sigma$ uncertainties of the line fitting. The narrowing parameters deduced from both measurements agree mostly within $2 \sigma$. There is a clear correlation between $\gamma_{2}$ air and $\gamma_{\text {air }}$ with $\gamma_{2 \text { air }}$ being smaller by a factor 4 to 7 .
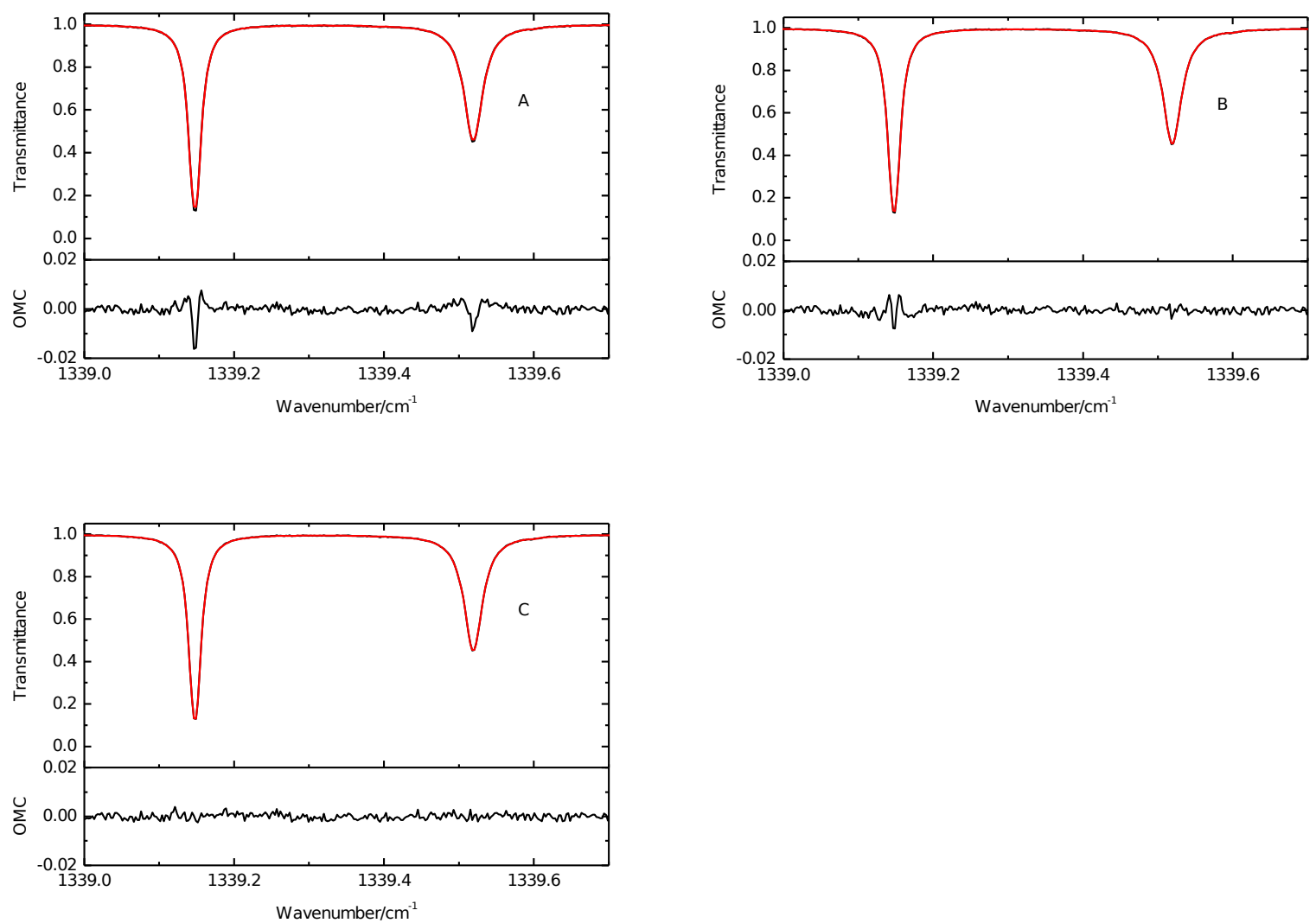

Figure 10. Different fits of two lines in the $\mathrm{H}_{2} \mathrm{O} v_{2}$ fundamental (left: $6_{6,0} \leftarrow 7_{7,1}$, right: $6_{4,2} \leftarrow 7_{5,3}$ ). Observed black, calculated red, observed-calculated in lower panels. Line positions, line intensities and quadratic baseline were fitted in all cases. Experimental conditions for observed spectrum see Figure 11, red. A: Voigt profile in line fitting, Lorentz broadening fixed to $\gamma_{\text {air,new. }}$ B: Voigt profile in line fitting, Lorentz broadening fitted. C: SDVP in line fitting, Lorentz broadening fixed to $\gamma_{\text {airnew }}, \gamma_{2}$ air fitted. 


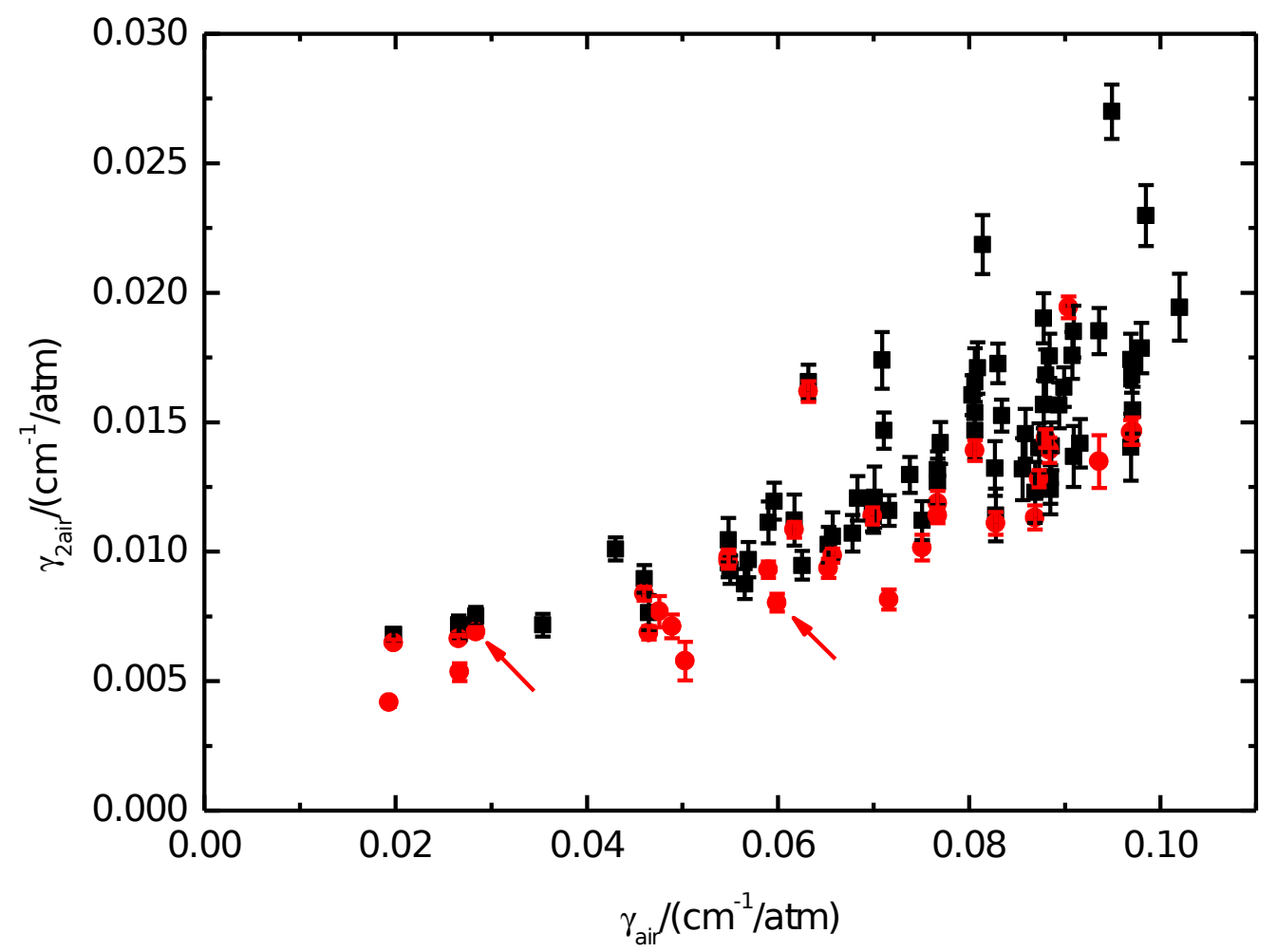

Figure 11. Narrowing parameter vs. air broadening parameter. Black: $P_{\text {H2O }} 0.024$ mbar, $P_{\text {tot }}$ $\left(\mathrm{H}_{2} \mathrm{O}\right.$ +air) $200 \mathrm{mbar}$, absorption path $79 \mathrm{~m}$, temperature $296 \mathrm{~K}$. Red: $P_{\mathrm{H} 2 \mathrm{O}} 0.20 \mathrm{mbar}, P_{\text {tot }}$ $\left(\mathrm{H}_{2} \mathrm{O}+\right.$ air $) 200$ mbar, absorption path $21 \mathrm{~m}$, temperature $296 \mathrm{~K}$.

An alternative way to prove that narrowing (Dicke or speed-dependence) together with Voigt fitting of non-opaque lines is causing saturated lines to be modelled too narrow would be a multispectrum fit of optically thin and saturated lines together, applying a narrowing profile. In case residuals which are present for a Voigt fit vanish for the narrowing profile the proof is given. At the time of this investigation the DLR multispectrum fitting tool was not yet ready and thus another route was taken. To our knowledge none of the groups routinely using multispectrum fitting has stumbled over the problem of opaque lines represented by effective Lorentz broadening. Furthermore they were apparently not aware of the advantage in combining non-opaque and opaque lines in order to gain sensitivity for determining narrowing parameters.

\section{Impact}

The present investigation is limited to parts of the $v_{2}$ fundamental of water. Furthermore, narrowing data in the literature are rather rare. Thus, the impact on other molecules or spectral regions is unknown. The findings in the present paper have importance for the following areas: 
Remote sensing. Strong lines in atmospheric spectra are modeled systematically too narrow when using a spectroscopic database derived applying the Voigt profile. Thus, systematic errors in the retrieved profiles are likely, especially in case of NADIR instruments like IASI [26] requiring strong lines for temperature sounding. Indeed, valleys between strong lines in IASI spectra are modeled too deep [16].

Laboratory spectroscopy. In order to provide correct spectroscopic data it is important to measure collisional narrowing parameters with an appropriate line profile. So far these data are rather sparse in the literature. The new results presented in this paper allow an effective way of determining collisional narrowing by multispectrum fitting of saturated and non-saturated lines simultaneously. Theoreticians should be aware when comparing models to experimental values that the Voigt parameters in literature are systematically too small when ignoring narrowing.

Climate modeling. Top of the atmosphere radiative fluxes and $\mathrm{CO}_{2}$ radiative forcing are dependent on the spectroscopic databases. Kratz [27] has calculated that radiative forcing by simultaneous doubling of $\mathrm{CO}_{2}, \mathrm{CH}_{4}$, and $\mathrm{N}_{2} \mathrm{O}$ (taken from Table 6 of [27]: -2.4 W/m² subarctic winter, $-4.8 \mathrm{~W} / \mathrm{m}^{2}$ tropics) changes by up to $5 \%$ when changing the database from HITRAN 82 to 2004. FasCODE [28] calculations resulted in $-0.4 \mathrm{~W} / \mathrm{m}^{2}$ change in mid-latitude summer top of the atmosphere radiative flux when increasing pressure broadening of $\mathrm{H}_{2} \mathrm{O}$ by $10 \%$. Of course, optically thin lines do not affect radiative forcing when increasing pressure broadening since the integrated absorption is only depending on the line intensity, thus only the opaque lines are contributing to the $-0.4 \mathrm{~W} / \mathrm{m}^{2}$. Assuming all opaque lines to become $3.5 \%$ broader, corresponding to our findings, the forcing by simultaneous doubling of $\mathrm{CO}_{2}, \mathrm{CH}_{4}$, and $\mathrm{N}_{2} \mathrm{O}$ would be increased by $3 \%$. These numbers indicate that the systematic linewidth error may be of significance.

Continua. In the experimental determination of continua the difference of a measured and modelled spectrum is formed where the modelling is usually based on the Voigt profile [17] [29]. The systematic error of saturated lines being modelled too narrow may distort the resulting continuum. For investigation spectra were modelled for conditions as used by Paynter et al. [29] to determine the foreign continuum in the $v_{2}$ region. Two spectra were modelled with HITRAN2012 line parameters where for one of them air broadening parameters were increased by $3.5 \%$ for lines with optical depth $>4$. Pseudo continuum absorption cross sections were calculated from the ratio of the transmittances quantifying the influence of the spectroscopic parameter change on the continuum for this specific measurement scenario. This error spectrum shows a spectral shape different from the continuum and maximum values of about $10 \%$ of the continuum. Increasing the air broadening parameter of all lines yields a very similar error spectrum which is expected since errors in weaker lines have less impact on the continuum. Regarding the uncertainty in the continuum (see error bars in Figure 15 in [29]) the resulting $10 \%$ systematic error is small. Anyhow, in order to avoid this systematic error contribution continuum calculations should be carried out with a SDVP.

The effect of too narrow opaque lines on water vapor foreign continuum retrieval between 1200 and $1800 \mathrm{~cm}^{-1}$ was simulated with conditions taken from [29]. The maximum error was found to be $>10 \%$ for $3.5 \%$ Lorentz width decrease. The effect on other continua cannot be quantified now due to the lack of data. Anyhow it is desirable to derive continua with the proper line profile. 


\section{Conclusion}

The present investigation has shown that line narrowing is the cause of systematic errors between measured and calculated saturated lines observed when the spectroscopic database is based on the Voigt line profile. The problem was discovered in air broadened measurements of the $\mathrm{H}_{2} \mathrm{O} v_{2}$ fundamental. Opaque lines can be fitted with the Lorenz function down to the noise level when the line intensity is fixed in the fit. The values are about $3-4 \%$ larger than the effective broadening parameters which are in the spectroscopic databases obtained from non-opaque lines. The problem arising from the Voigt function is avoided by using a line profile covering narrowing effects which in turn requires the availability of narrowing parameters together with their temperature dependence. The combination of saturated and non-saturated measurements provides a very sensitive method for determining narrowing parameters avoiding the need for high signal-to-noise measurements. Furthermore, opaque lines are suited for line shape investigations. The results of the present work have impact on remote sensing and possibly climate modelling.

In order to avoid the systematic error presented here narrowing parameters must be determined for key atmospheric species and entered into spectroscopic databases. Remote sensing groups should use narrowing profiles (equivalent to that for laboratory analysis) and redo analysis.

\section{Acknowledgements}

The authors thank Shepard A. Clough for triggering this work and his critical comments in the first phase of manuscript preparation and Joep Loos for critically reading this manuscript. We acknowledge BMBF support in the frame of the HGF-Vernetzungsfonds "Generierung und Validierung von Datenprodukten aus ENVISAT-Messungen sowie deren Nutzung zur Erforschung der oberen Troposphäre und Stratosphäre” and support by the WDC-RSAT at DLR.

\section{References}

[1] Roeckner E, Bäuml G, Bonaventura L, Brokopf R, Esch M, Giorgetta M, et al. The atmospheric general circulation model ECHAM5. PART I: Model description. Tech. Rep. 349, Max-Planck-Inst. für Meteorol., Hamburg, Germany, 2003.

[2] Bernath PF, McElroy CT, Abrams MC, Boone CD, Butler M, Camy-Peyret C, et al. Atmospheric chemistry experiment (ACE): mission overview. Geophys Res Lett 2005;32:L15S01.

[3] Fischer H, Birk M, Blom C, Carli B, Carlotti M, von Clarmann T, et al. MIPAS: An Instrument for Atmospheric and Climate Research. Atmos Chem Phys 2008;8:2151-88.

[4] http://www.ndsc.ncep.noaa.gov/

[5] Rothman LS, Rothman IE, Barbe A, Benner CD, Bernath PF, Birk M, et al. The HITRAN 2008 molecular spectroscopic database. J Quant Spectrosc Radiat Transfer 2009;110:533-72, doi:10.1016/j. jqsrt.2009.02.013. 
[6] Jacquinet-Husson N, Scott NA, Chedin N, Crépeau A, Armante R, Capelle V, et.al. The GEISA spectroscopic database: Current and future archive for Earth and planetary atmosphere studies. J Quant Spectrosc Radiat Transfer 2007;109:1043-59, doi: 10.1016/j.jqsrt.2007.12.015.

[7] Olivero JJ, Longbothum RL, Empirical fits to the Voigt line width: A brief review. J Quant Spectrosc Radiat Transfer 1977;17:233-6.

[8] Ciurylo R, Shapes of pressure- and Doppler-broadened spectral lines in the core and near wings. Phys Rev 1998;58:1029-39.

[9] Birk M, Wagner G, Temperature-dependent air broadening of water in the $1250-1750 \mathrm{~cm}-1$ range. J Quant Spectrosc Radiat Transfer 2012;113:889-928, doi:10.1016/j.jqsrt.2011.12.013.

[10] Duchatelet P, Demoulin P, Hase F, Ruhnke R, Feng W, Chipperfield MP, Bernath PF, Boone CD, Walker KA, Mahieu E. Hydrogen fluoride total and partial column time series above the Jungfraujoch from long-term FTIR measurements: Impact of the line-shape model, characterization of the error budget and seasonal cycle, and comparison with satellite and model data. J Geophys Res 2010;115:D22306, doi:10.1029/2010JD014677.

[11] Barret B, Hurtmans D, Carleer MR, De Mazière M, Mahieu E, Coheur P-F. Line narrowing effect on the retrieval of $\mathrm{HF}$ and $\mathrm{HCl}$ vertical profiles from ground-based FTIR measurements. J Quant Spectrosc Radiat Transfer 2005;95:499-519, doi:10.1016/j.jqsrt.2004.12.005.

[12] Schneider M, Hase F, Improving spectroscopic line parameters by means of atmospheric spectra: Theory and example for water vapor and solar absorption spectra, J Quant Spectrosc Radiat Transfer 2009;110:1825-39.

[13] Coudert LH, Wagner G, Birk M, Baranov YI, Lafferty WJ, Flaud JM. The $\mathrm{H}_{2}{ }^{16} \mathrm{O}$ molecule: line position and line intensity analyses up to the second triad. J Mol Spectrosc 2008;251:339-57.

[14] Anderson GP, Clough SA, Kneizys FX, Chetwynd JH, Shettle EP. AFGL atmospheric constituent profiles (0-120 km). Rep. AFGL-TR-86-0110, Air Force Geophys. Lab., Hanscom Air Force Base, Bedford, Mass. 1986.

[15] Rothman L, Gordon I, Babikov Y, Barbe, A, Benner, D Chris, Bernath, PF, et al. The HITRAN 2012 Molecular Spectroscopic Database. J Quant Spectrosc Radiat Transfer 2013;130:4-50, doi: 10.1016/j.jqsrt.2013.07.002.

[16] Clough SA. private communication, 2012.

[17] Clough SA, Kneizys FX, Davies RW. Line Shape and the Water Vapour Continuum, Atmos Res 1989;23:229-41.

[18] Dicke RH. The Effect of Collisions upon the Doppler Width of Spectral Lines. Phys Rev 1953;89:472-3, doi:10.1103/PhysRev.89.472.

[19] Berman PR. Speed-dependent collisional width and parameters in spectral profiles. J Quant Spectrosc Radiat Transfer 1972;12:1331-42. 
[20] Ward J, Cooper J, Smith EW. Correlation effects in the theory of combined Doppler and pressure broadening - I. Classical theory. J Quant Spectrosc Radiat Transfer 1974;14:555-90.

[21] Boone CD, Walker KA, Bernath PF. Speed-dependent Voigt profile for water vapor in infrared remote sensing applications. J Quant Spectrosc Radiat Transfer 2007;105: 525-32, doi:10.1016/j.jqsrt.2006.11.015.

[22] Hodges JT, Lisak D, Lavrentieva N, Bykov A, Sinitsa L, Tennyson J, Barber RJ, Tolchenov RN. Comparison between theoretical calculations and high-resolution measurements of pressure broadening for near-infrared water spectra. J Molec Spectrosc 2008;249:86-94, doi:10.1016/j.jms.2008.02.022.

[23] Lisak D, Havey DK, Hodges JT. Spectroscopic line parameters of water vapor for rotation-vibration transitions near $7180 \mathrm{~cm}^{-1}$. Phys Rev A 2009;79:052507, doi: 10.1103/PhysRevA.79.052507.

[24] Miller CE, Brown LR, Toth RA, Benner DC, Malathy Devi V. Spectroscopic challenges for high accuracy retrievals of atmospheric $\mathrm{CO}_{2}$ and the Orbiting Carbon Observatory (OCO) experiment. C R Physique 2005;6:876-887, doi:10.1016/j.crhy.2005.09.005

[25] Ngo NH, Tran H, Gamache RR, Hartmann JM. Pressure effects on water vapour lines: beyond the Voigt profile. Phil Trans R Soc A 2012;370:2495-2508, doi:10.1098/rsta.2011.0272

[26] Schlüssel P, Hultberg TH, Phillips PL, August T, Calbet X. The operational IASI Level 2 processor. Advances in Space Research 2005;36:982-8, doi:10.1016/j.asr.2005.03.008.

[27] Kratz DP. The sensitivity of radiative transfer calculations to the changes in the HITRAN database from 1982 to 2004. J Quant Spectrosc Radiat Transfer 2008;109:1043-59, doi:10.1016/j.jqsrt.2007.10.010.

[28] Clough SA, Kneizys FX, Shettle EP, Anderson GP. Atmospheric radiance and transmittance: FASCOD2. Proc. 6th Conf. Atmospheric Radiance. AMS, Williamsburg, VA, 141-4 (1986).

[29] Paynter DJ, Ptashnik IV, Shine KP, Smith KM, McPheat R, Williams RG. Laboratory measurements of the water vapor continuum in the $1200-8000 \mathrm{~cm}^{-1}$ region between $293 \mathrm{~K}$ and 351 K. J Geophys Res [Atmos] 2009;114:D21301, doi:10.1029/2008JD011355. 